\title{
HYDROFOILS AND HYDROFOIL CRAFT
}

\author{
A. J. Acosta \\ California Institute of Technology, Pasadena, California
}

\section{INTRODUCTION}

At the present time several hundred hydrofoil craft are in service throughout the world. The upsurge in the use of thesecraft did not really begin until the late 1950s, although fascination with the idea of supporting small boats with underwater wings dates well back into the nineteenth century. About the turn of the century, hydrofoil flight was achieved, to be followed in a few years by Bell and Baldwin who, at the close of World War I, achieved the modern hydrofoil speed of 60 knots in a very novel craft. Progress and interest in this form of transportation then waned for many years. There were some interesting developments just prior to and through World War II in Europe, and after the war interest quickened in several countries. Current thinking at this time may be judged by Gabrielli \& von Kármán (1950), who note that the drag of surface vessels may be decreased by lifting the floating structure with hydrofoils. But they go on to add that they "... do not attempt to estimate the effect of such a radical innovation; whether the trials until now appear promising is a question of individual judgment." As subsequent events have proven, the trials were indeed promising. The many hydrofoil craft now in service are of several different types and a number of more advanced concepts are being developed swiftly.

Greater speed in all forms of transportation has always been sought, provided the price is not too great. The air-sea surface is a particularly inhospitable environment for major advances in operating speed, yet it is partly this advance that is the stimulus for hydrofoil craft as well as that for conventional marine craft and the newer hover craft. Silverleaf (1970) and Silverleaf \& Cook (1970) review all these recent developments from technical and economic standpoints. High speeds at sea are now possible, they observe, but may not be attained for lack of naval or commercial demands.

The successful achievements of hydrofoil craft to date and the possibility of high speeds at sea are due to the greatly increased understanding in recent years of the flow past hydrofoils and also to the development of foil configurations and control systems for coping with the roughness of the sea surface. It seems appropriate, therefore, in this review to link the discussion of hydrofoils with that of 
progress in the craft, for the two are very interdependent. This interaction has been the source of a great deal of research in applied fluid mechanics in recent years. The methods of analysis, experiment, and design in this field follow closely those in aeronautics, yet there are some important differences because of the medium itself. These include the phenomena due to the free surface (an everpresent boundary in naval hydrodynamics) and the possibility of a phase change through cavitation or ventilation with consequent important modification of the flow; because the liquid density is so much greater than in equivalent aeronautical applications. the dynamic response to motions must be treated very carefully. Hydrofoils, in addition, find application as control surfaces and structural members in marine craft generally, and serve also as elements of propulsion devices.

In what follows, the general characteristics of hydrofoil craft are briefly reviewed together with some representative modern examples. Some physical aspects of the flow past hydrofoils are then described, followed by a résumé of some of the recent methods used in design and analysis of hydrofoils. In this short account several important topics have of necessity been omitted; these include propulsion, hydroelastic problems, and ship motions, each of which could serve as the topic of a separate article.

\section{HYDROFOIL CRAFT}

\section{General Features}

A hydrofoil craft has two modes of operation: the normal slow-speed hullborne mode and, with increasing speed through the takeoff, the flying or foilborne mode. Takeoff may occur at speeds near the maximum hull-borne drag or "hump" speed with subsequent acceleration to the cruise speed, which typically may be twice the takeoff speed. The drag-speed characteristic of foil craft has a broad minimum between hump speed and cruise speed beyond which resistance begins to climb rapidly (see, e.g., Krack \& Gross 1965 , Crewe 1958). In the foilborne mode the effective lift-drag ratio must be adequate for the intended operation, and at the same time the craft must be able to fly in a stable, controllable manner with a satisfactory environment for crew and/or passengers. These desiderata are somewhat conflicting and the contributing factors are very speed. dependent. As a result, a number of designs have emerged which with various weights on economic factors have emphasized differently or provided different solutions to these general requirements.

\section{Foil Systems}

Many different configurations are possible although there must always be lifting surfaces forward and aft for longitudinal stability. The load may be divided more or less evenly between these foils in a tandem arrangement, or most of the load may be carried on the bow foil in what is termed an airplane or conventional configuration. Alternatively, the aft foil may support most of the weight in the 
canard configuration. Each of these fore or aft lifting surfaces may be split into two independent foils for purposes of design or for retraction in shallow water. There is a basic distinction between different types of craft in the method used for stabilization and control once foil-borne operation is achieved and in obtaining the modulation of dynamic lift needed between takeoff and cruise. An early and still successfully used principle is that of variable-area stabilization with surfacepiercing hydrofoils. These hydrofoils may consist of a single member in the shape of a V or W or of several members arranged in a cascade or ladder. Requirements of takeoff and stability can be satisfied with either arrangement although the resulting system is sensitive to waves. An alternate scheme is to submerge both lifting surfaces, avoiding thereby area changes with changes of relative waveheight. Submerged-foil as well as surface-piercing systems experience orbital wave motions which may be appreciable, and because the lifting-surface area is fixed, this system is inherently less stable. All of these arrangements are in present use. Lift modulation with flaps, variable incidence, or spoilers provides additional means of control, trim, and load alleviation and is employed on both surface-piercing and submerged-foil systems of recent design.

\section{Limiting Factors}

Dynamic pressure reduction of $\sim 1 \mathrm{~atm}$ on the foils of surface craft leads to the phenomenon of cavitation. This does not necessarily result in adverse effects on performance; if the extent of cavitation is small there is little effect except for the possible occurrence of erosion, but when the region affected is comparable to the chord or larger, major changes in lift and drag result. This, with sufficient margins for control, interaction with other components, and the possibility of ventilation of surface air to the lifting surface, limits foil loadings to $\sim \frac{1}{2}$ atm (Ellsworth 1967), about an order of magnitude greater than that for aircraft despite the density factor of 3 orders of magnitude. This has the result that design lift coefficients tend to be low (about 0.3 at 40 knots) and to decrease with increasing speed. A further and major obstacle is the sea surface itself, which has to be negotiated. At low speeds this surface may be easily contoured; human sensitivity to oscillating vertical motion is such that a pronounced discomfort is produced at about $0.1 \mathrm{~g}$ acceleration levels in the frequency range of $0.1-2 \mathrm{~Hz}$. With the frequency of wave encounter to be expected for a significant waveheight of only $4 \mathrm{ft}$ (Oakley 1963, Tulin 1960) at a speed of 40 knots this requires the heave amplitude to be less than $0.1 \mathrm{ft}$. For all practical purposes the craft must "platform" through waves, if incapacitating discomfort is to be avoided. Much of the thrust in the development of hydrofoil craft has been directed towards minimizing and reducing the effects of these wavemotions (see, for example, French et al 1962, Hirsch 1967, Martin 1967, for representative dynamic studies).

\section{Hydrofoil Developments}

The first marked success in hydrofoil development is due to Forlanini, who in 1906 demonstrated a craft displacing 1.65 tons that achieved a speed of 38 knots 
with a 75-hp engine (Crewe 1958), which compares favorably with modern craft. Forlanini used a ladder-foil system having zero dihedral (rather like a venetian blind), so that heave stability over the speed range was inherent. At about the same time, other workers were interested in applications to seaplanes, among whom may be mentioned the Wright brothers. Remarkable for its foresight is the fully submerged hydrofoil system patented by Meacham in 1906. In this device, planing surfaces linked to submerged foils control the height of the craft. A more modern version of this type of arrangement, employing mechanical sensors, is Hook's Hydrofin. Of these early efforts, that of Bell and Baldwin (J. H. Parkin 1964) is most notable; they employed a three-point support in the airplane configuration of ladder foils having some dihedral; air propelled, the maximum lift drag ratio of the foils is estimated to be 7-8. Their fastest run on September 9, 1919, of 61.6 knots with their craft HD-4 is still hardly exceeded even by modern hydrofoil craft. Other contemporary workers were still concerned with hydrofoil-assisted seaplane takeoff, just as Bell and Baldwin were to begin with. None of these early activities, including Bell's, ever came to fruition in a commercial or naval craft.

The next major steps were made by von Schertel (1963), who between 1927 and 1937 developed the tandem surface-piercing foil system, which subsequently was pursued intensively by the Supramar firm of Switzerland during the early 1950s. In wartime years, several craft of this type were developed as well as a fast (55 knot) craft having a surface-piercing front foil and fully submerged after foil, due to O. Tietjens (Crewe 1958), whose name will be familiar to readers of these reviews.

After the war, the development of a number of different concepts was supported in the United States by the Department of the Navy and the Maritime Administration (Buerman et al 1953, Oakley 1963). Noteworthy of this period was the development of fully submerged-at first mechanically, then later actively -stabilized foil systems (Ellsworth 1967) and the emergence of fully cavitating or "supercavitating" hydrofoil and propeller technology (Cooper 1958, Anon. 1958). By this time, sufficient information was available to make engineering system studies of large and extremely fast craft (Geyer \& Wennagel 1959). Although recent emphasis has ultimately come to be centered on fully submerged, actively controlled systems, surface-piercing foil craft were not neglected (Krack \& Gross 1965). Further developments of surface-piercing craft, which can actually be traced back to the original Bell-Baldwin work, continued in Canada, to result in the 200-ton, 60-knot naval hydrofoil ship Bras-D'Or (Eames 1970). Although it was clear that surface-piercing foil systems had inherent stability, until recently it was believed that submerged foils required automatic lift control to obtain stabilization (see, e.g., von Schertel 1960). It is known, however, that as a foil approaches the water surface the lift force in fully wetted flow decreases; depending upon aspect ratio this decrease may be one-half the fully submerged value (Schuster \& Schwaneke 1960). This surface-proximity effect is the basis of a highly successful class of hydrofoil craft developed in the Soviet Union (McLeavy 1971) for service in protected waters. Fixed foils are arranged in tandem; in one design the front foil is split, and auxiliary foils attached to the bow-supporting 
Table 1 Physical characteristics of representative hydrofoil craft

\begin{tabular}{|c|c|c|c|c|c|}
\hline Vessel & $\begin{array}{l}\text { Displacement } \\
\text { (tons) }\end{array}$ & $\begin{array}{l}\text { Length } \\
\text { (ft) }\end{array}$ & $\begin{array}{l}\text { Power } \\
\text { (hp) }\end{array}$ & $\begin{array}{c}\text { Speed } \\
\text { (knots) }\end{array}$ & Type \\
\hline 1. Tucumcari (USA) & 57.5 & 74.6 & 3,200 & $>45$ & $\begin{array}{c}\text { Canard, split, } \\
\text { water jet }\end{array}$ \\
\hline 2. Plainview (USA) & 320 & 219.4 & 28,000 & $>50$ & $\begin{array}{l}\text { Airplane, split, } \\
\text { s/c prop. }\end{array}$ \\
\hline 3. PTS $150 \mathrm{MK}$ III (Switz.) & 165 & 124.2 & 6,880 & 36.5 & Tandem, prop. \\
\hline 4. $V i k h r($ USSR) & 117.5 & 156 & 4,800 & $\begin{array}{r}35.8 \\
\text { (cruise) }\end{array}$ & Tandem, prop. \\
\hline
\end{tabular}

struts assist in takeoff. Some other Soviet craft are more similar to the Supramar designs, having a surface-piercing $\mathrm{V}$ bow foil, but a more recent development employs fully submerged foils with automatically controlled flaps for service in moderate seas at speeds of 38-42 knots.

\section{Representative Craft}

The principal characteristics of some recent hydrofoil craft are shown in Table 1, each craft being of quite a different configuration: Craft 1 is water-jet propelled and has split main foils in canard configuration. Inertial and height sensors provide inputs for full automatic control of hydraulically actuated flaps. Normally all turns are fully coordinated. Craft 2 , a large research vessel, has fully retractable, split main foils in airplane configuration, as can be seen in Figure 1. Propulsion is by supercavitating propeller; stabilization is achieved by hydraulic

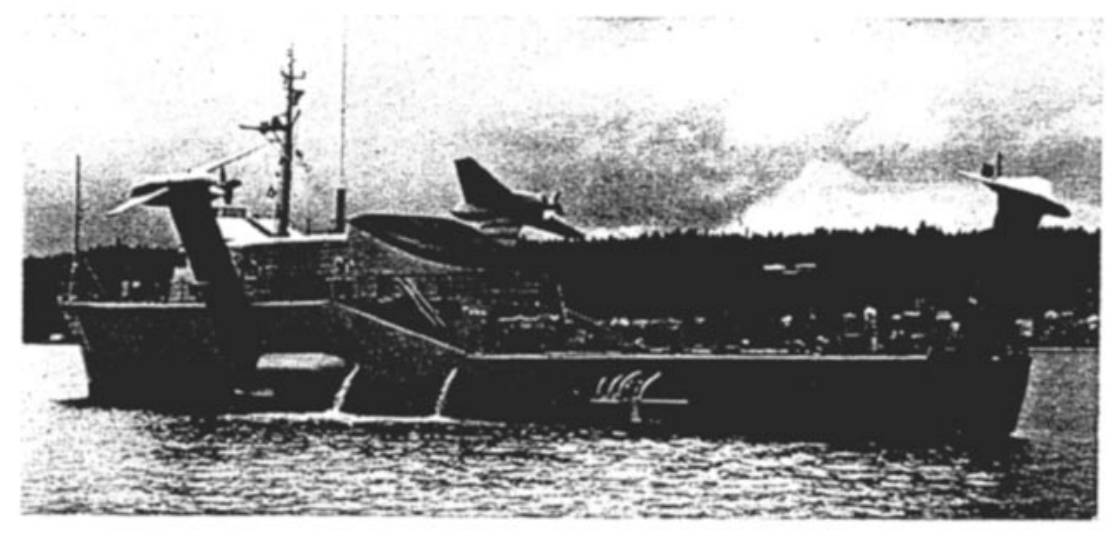

Figure 1 Photograph of USS Plainview with wings retracted showing airplane foil configuration and supercavitating propellers. The wing span of the mainfoils is $26 \mathrm{ft}$ (Courtesy Naval Ship Research and Development Center.) 
incidence control of the main foils. Craft 3, presently the largest commercial foil craft in service, is the most recent development of the Schertel system with a surface-piercing bow foil fitted with trailing-edge flaps for trim adjustment. The stern foil, fully submerged, is controlled by a novel system of lift spoiling by introduction of air into regions of low pressure. The deliberate (and successful) use of "ventilation" to modify lift on fully wetted hydrofoils has been elaborated by Lang \& Daybell (1961), and a patent employing this principle has been awarded (Smith 1963). The air ventilation or "bleed" method avoids physical actuation of flaps or foils and appears to be a very useful scheme capable of considerable development for the generation of control forces and load alleviation in waves (von Schertel 1970), although with some increase in drag. The last example, Craft 4 (Vikhr), employs submerged bow and stern foils with an additional amidship foil, is said to have some form of stability augmentation, and is suitable for operation in coastal waters having moderate seas.

\section{Overall Performance}

A figure of merit that reflects overall hydrodynamic and propulsion performance is the "transport efficiency" $\eta L / D=W V / P$, where $\eta$ is the effective propulsive efficiency, $L / D$ the lift-to-drag ratio, $W$ the displaced weight, $V$ the craft speed, and $P$ the absorbed power in consistent units. This ratio, useful in comparing similar types of craft, is presented in Figure 2 based on installed power. The abscissa is the Froude number $F=V(g \ell)^{-1 / 2}$ based on the length $\ell=(W / \rho g)^{1 / 3}$ in consistent units, where $\rho$ is the fluid density and $g$ the acceleration of gravity. Three classes of craft are represented in this diagram. The advantage of the hydrofoil with increasing speed over high-speed displacement boats is evident (except for the overpowered Plainview). Amphibious hovercraft are seen to be higher-speed craft but even in a moderate sea suffer a major reduction in speed due not only to greater hydrodynamic drag but also because of the necessity to reduce ship motion to a tolerable level. Displacement craft are even more at a disadvantage in an appreciable seaway. Submerged hydrofoil craft with liftalleviation systems do not suffer the disadvantage of being closely coupled with the water surface and accordingly can achieve low levels of vertical acceleration of approximately $0.1 \mathrm{~g}$ or less, which is required to avoid motion sickness and discomfort (Silverleaf \& Cook 1970, von Schertel 1972, Schultz et al 1971). These effects combine to produce the dimunition in speed with waveheight shown in Figure 3, although the vertical accelerations experienced by the displacement, amphibious, and surface-piercing craft may still be several times larger than for the fully submerged, stabilized foil craft.

Transport efficiency has been given as a function of speed coefficient in Figure 2 , as this is a conventional parameter in naval hydrodynamics, used to represent the effect of gravity in wave-making resistance. This is a contributing but small effect for hydrofoil craft except near takeoff, when the resistance of the main hull is important. More important is the effect of speed itself, as this determines the basic configuration and relation between design parameters, the nccurrence 


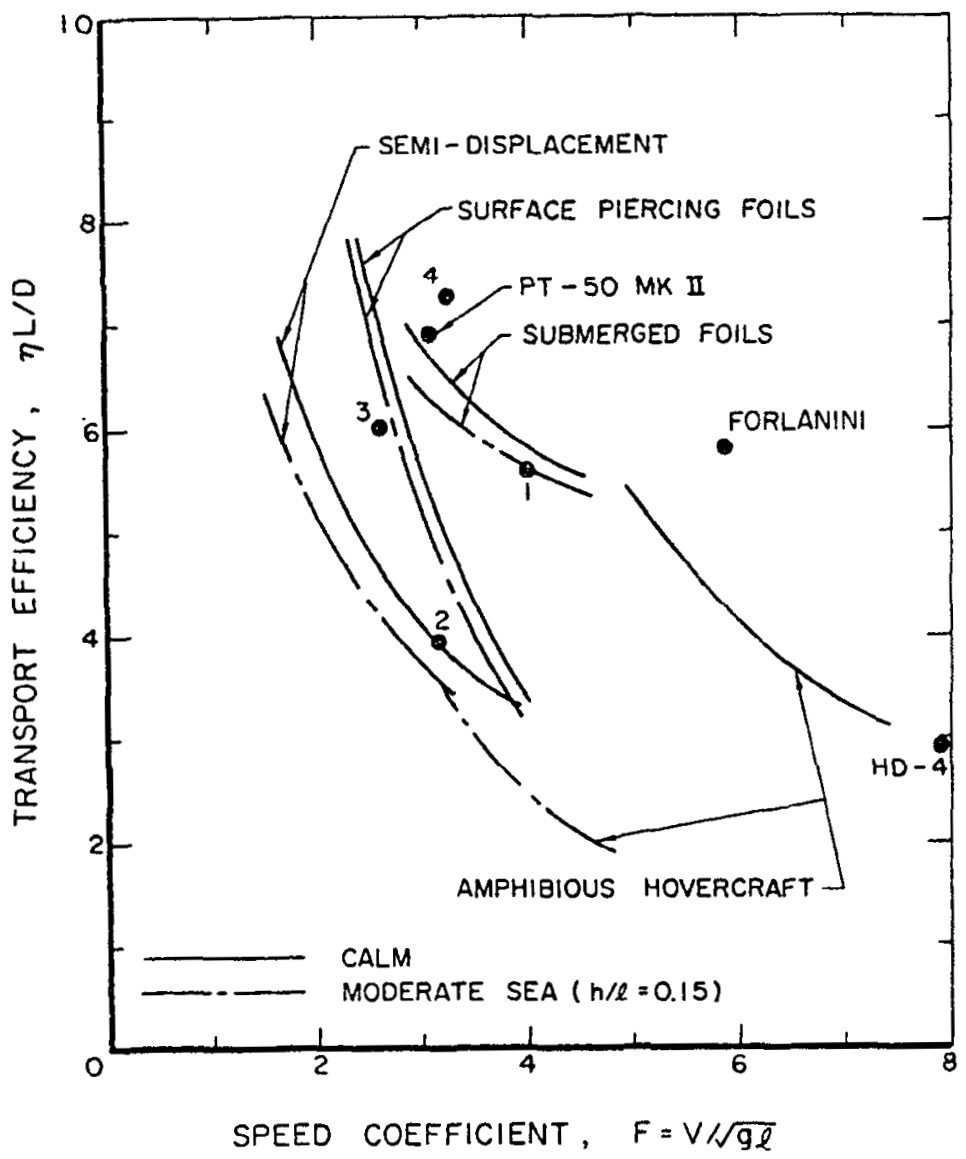

Figure 2 Transport efficiency $\eta L / D \equiv W V / P$ vs speed coefficient $V(g l)^{-1 / 2}$, for several types of high-speed craft (after Silverleaf and others). In this, $W$ is the displaced weight, $V$ the speed, $P$ the installed power, $g$ the acceleration of gravity, and $\rho$ the fluid density; $\ell=(W / \rho g)^{1 / 2}$ all in consistent units; $h$ is the wave height for a moderate sea condition. The numbers refer to the craft listed in Table 1.

of cavitation, and possibly unwanted air ventilation of the lifting surfaces. Except for waves, variable-area systems could operate ideally at constant liftdrag ratios until the usually adverse effects of cavitation/ventilation commence, but submerged foil systems are at a disadvantage in this respect. Beyond speeds of about 50 knots, cavitation becomes increasingly difficult to avoid. All these considerations, together with the increasing problems of propulsion with advanced speed, have meant speed-limited performance. Thus it is that the over- 


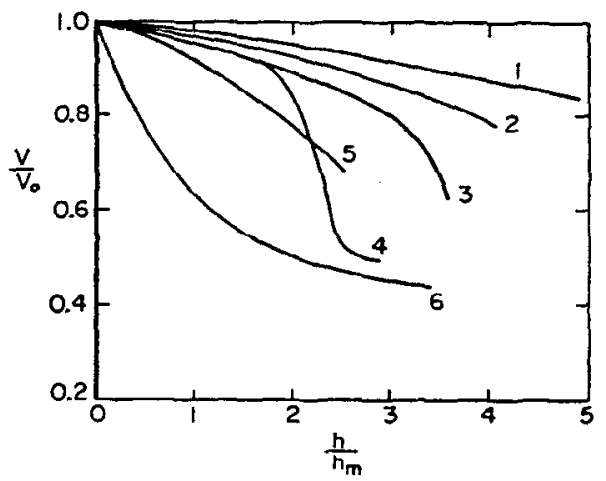

Figure 3 Reduction of sustained speed with waveheight for various types of craft (after Silverleaf and others). Curve (1) refers to submerged hydrofoil craft, (2) airstabilized submerged foil craft, (3) semi-surface-piercing craft, (4) surface-piercing craft, (5) semi-displacement craft, and (6) amphibious air-propelled hovercraft; $h_{m}$ is the waveheight for a moderate sea $(=0.15 \ell)$.

whelming majority of commercial hydrofoil craft have service speeds of about 38 knots or less. At speeds somewhat higher than this all known craft begin to exhibit a sharply lower transport efficiency. Next, we consider some of these effects in more detail.

\section{TWO HYDRODYNAMIC PHENOMENA}

We now consider briefly two phenomena of major importance for hydrodynamics, cavitation and ventilation. Cavitation is said to occur when through dynamic pressure reduction via the Bernoulli effect the vapor phase of the liquid is formed. Ventilation is said to occur when a noncondensible gas such as air forms attached wakes or "cavities" on submerged bodies in a flow. In either case the flow field exhibits two discrete phases, the configuration of which except in certain simple cases cannot be predicted in advance; it follows that these subjects must be treated primarily experimentally.

\section{Cavitation}

Before the end of the last century Parsons became aware that cavitation was responsible for the poor performance of the propellers on his steamship Turbinia, and it is only comparatively recently that the problems introduced by this physical process have been coped with at all successfully. We are concerned in the present discussion with only one aspect of cavitation-its effect on the flow past hydrofoils (for this and related matters, see Knapp, Daily \& Hammitt 1970). 
This may be characterized into three stages: the onset or "inception" stage (of little importance for forces), a "partial" cavitation stage in which the vapor forms an attached "cavity" shorter than the chord, and a fully cavitating or "supercavitating" stage in which the vapor cavity is distinctly longer than the chord. In an obvious analogy, a fully wetted, noncavitating flow is sometimes called subcavitating flow. The principal physical parameter governing these flows is the pressure coefficient $k=\left(p_{0}-p_{0}\right) / q_{0}, q_{0}$ being the upstream dynamic pressure, $p_{0}$ the corresponding static pressure, and $p_{c}$ the pressure within the cavitating zone. This pressure may be greater or less than the hydrodynamic vapor pressure $p_{v}$ (often used in lieu of $p_{c}$ ), depending upon the fluid and the amount of dissolved gas (Knapp et al 1970), and if there is a possibility of ventilation it is likely that the two pressures are quite different. Even in the case of a closed water-tunnel experiment, considerable error may be made by assuming $p_{c}=p_{v}$ due to the diffusion of dissolved air (see e.g., Parkin \& Kermeen 1957).

The beginning of cavitation signifies the start of a new type of flow and raises the possibility of material damage through cavitation erosion. Therefore, in some applications it may be desirable to avoid any occurrence whatsoever of cavitation. Bodies having small values of $\left|-c_{p \min }\right|, c_{p}=\left(p-p_{0}\right) / q_{0}$, are therefore favored, with representative profile sections leaning towards the NACA 16 series thickness form with rooftop meanlines. Breslin \& Landweber (1961) summarize methods of calculating minimum pressures on two-dimensional hydrofoils, axisymmetric bodies, and junctures of two bodies in plane flow based on potential flow; such calculations provide useful but conservative estimates of cavitation inception. With the same objective in mind, Parkin \& Peebles (1956) outline procedures for the calculation of noncavitating hydrofoils with a prescribed pressure distribution.

The subsequent development of cavitation beyond the inception stage is very complicated, depending upon all of the factors attendant upon real-fluid twophase flows. These are touched upon in Wu's recent review (Wu 1972) and more extensively by Robertson \& Wislicenus (1969) and Eisenberg \& Tulin (1961). The general features of the process on hydrofoils are fairly well known (Walchner 1932, Numachi et al 1959, Wade \& Acosta 1966, Arakeri 1971). With decreasing pressure a vapor cavity becomes attached to the foil, usually somewhat downstream of the minimum pressure point. This may occur near the leading edge or, depending on the profile and angle of attack, downstream of the midpoint and in some cavities at both locations simultaneously. As a rule, partial cavitation is a highly unsteady and three-dimensional phenomenon, but under some conditions a "sheet-like" form of cavitation will originate from near the leading edge that has a stable smooth glassy surface. Ideal flow analyses (Wu 1972) then provide some information on the configuration of the cavity and the forces to be expected as a function of the cavitation number and geometry, provided the flow is two dimensional and the cavity detachment points are known. However, when the partial cavity is about a half chord in length or somewhat longer, the flow becomes unstable and a more or less periodic growth and collapse of the cavitation pattern is observed (Wade \& Acosta 1966). The force experienced by the 
Annual RePiew Costa

www.annualreviews.org/aronline

hydrofoil shows a similar unsteady behavior with an amplitude that may be an appreciable factor of the mean force (depending upon profile type). Once the terminus of cavity is about $1 / 3-$ to $1 / 2$-chord downstream of the trailing edge, the force on the hydrofoil becomes essentially steady. The behavior of the timeaveraged lift coefficient as a function of cavitation number for several different types of profile sections is indicated in Figure 4. Both the plano- and bi-convex foils have pointed leading edges, which gives rise to leading-edge partial cavitation and an initial increase of lift as cavitation develops. Partial cavitation on the NACA profile begins at midchord, spreading aft with decreasing cavitation numbers. Characteristically, the lift force decreases as this type of cavitation develops. It is worth noting that both the biconvex foils exhibit a small negative lift at

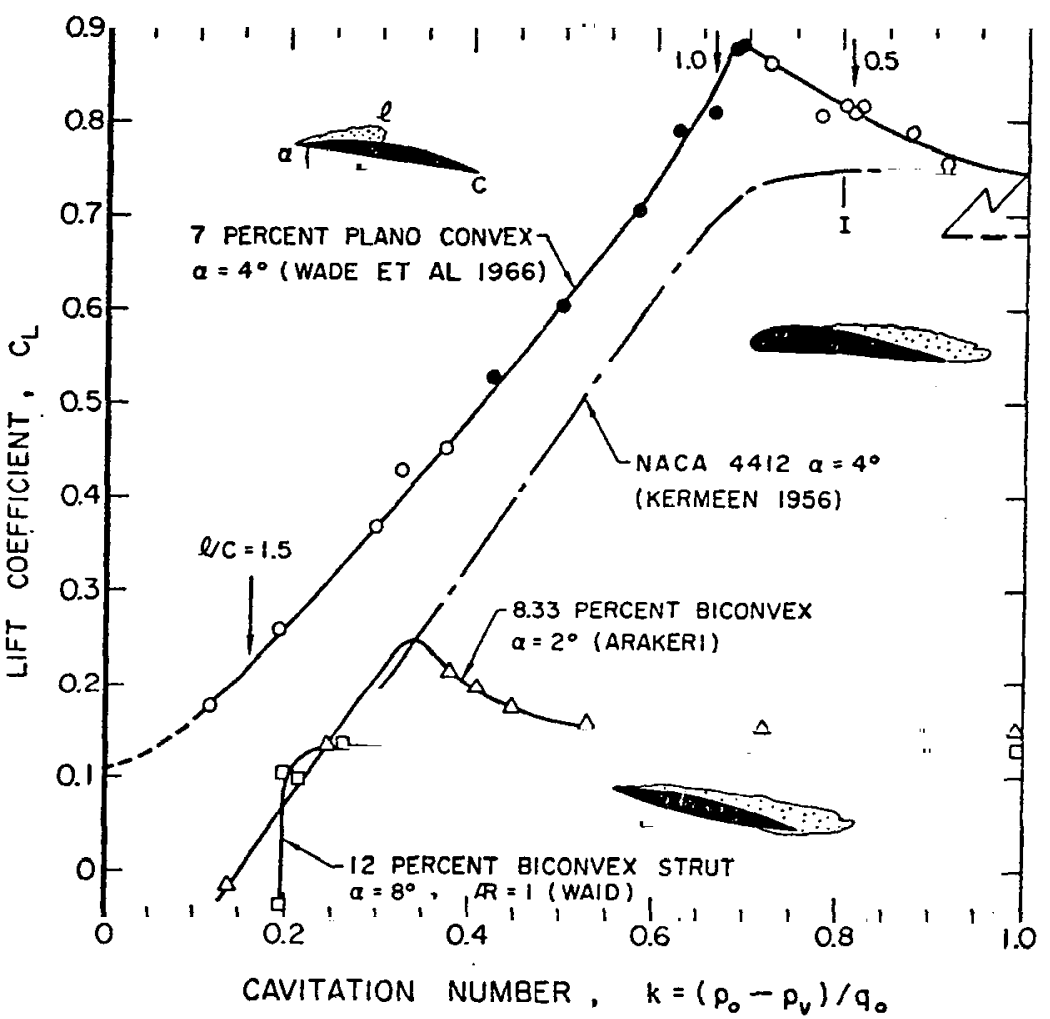

Figure 4 Lift coefficient $C_{L}$ vs cavitation number $k$ for several hydrofoils and struts. On the upper curve the numbers give the average length of the cavity; the solid points represent conditions of unsteady partial cavitation. The letter $l$ indicates the inception of cavitation on the NACA foil. 
positive angles of attack. In both cases this is due to the liquid flow remaining attached to the "pressure" side of the hydrofoil, which being convex to the flow produces negative lift. The $12 \%$ biconvex foil is a surface-piercing strut of aspect ratio unity. The sharp decrease of lift and subsequent development of negative lift is caused by ventilation from the surface-in this case triggered by the interaction between a large vapor cavity on the submerged portion of the foil and the nearby surface (Waid 1968). The approximate flow configuration of these foils in this case is sketched in the lower insert of Figure 4.

The development of cavitation has a profound influence on drag. Profiles having a generous leading-edge radius such as the NACA 4412 usually exhibit a severe increase in drag and decrease in lift-drag ratio with decrease in cavitation number (Kermeen 1956). Sharp-edged foils, however, typically show a decrease in drag in the initial-phase partial cavitation, with the corresponding reduction of wetted area in this mode of flow, without the increase of profile drag suffered by conventional foils derived from aerodynamic practice.

The course of the developments just described for single foils is generally observed in cascades of identical foils (Wade \& Acosta 1967, Numachi 1964). A different type of interaction with cavitation is possible for multiple foils where the mutual effect can be unsymmetric. For example, Egorov and co-workers (1971) show experimentally that the presence of a small staggered auxiliary foil (supercavitating) can suppress the development of partial cavitation on a main foil under conditions where the latter would normally be supercavitating. Otherwise, this type of problem seems to have received very little attention except for the analytic studies of Green \& Street (1967) on tandem supercavitating foils.

The development of the partial cavitation region and of unsteadiness of the flow and forces appears to be strongly influenced by aspect ratio and planform (King \& Land 1952, Kermeen 1961, Dobay 1965). An important feature of these flows is the strongly three-dimensional cavity that can develop at the tip. Finiteaspect-ratio supercavitating foils designed to achieve a smooth transition through the partial cavitation mode by spanwise development of the cavity have been proposed but apparently not tested (Ellsworth 1967). Again, as for two-dimensional sections, the supercavitating flow past finite planforms is essentially steady although, except for simple bodies, comparatively little experimental work has been reported (see, for example, Dobay 1967).

\section{Ventilation}

The process by which air or other noncondensible gas forms attached cavities on ;bodies in a flow is complicated and poorly understood except in a general way. The terminus of a ventilated cavity in most technical applications is at a sufficiently high Reynolds number so that gas is entrained away into the surrounding liquid flow; for such ventilation to be stable and permanent, replacement gas must be supplied at the same rate. When the replacement gas is supplied from the surface along paths afforded by air-filled wakes behind hydrofoils, struts, or trailing vortex cores of submerged lifting surfaces, one speaks of natural ventila- 
A Annual RkzZews Acosta

www.annualreviews.org/aronline

tion. However, as in the case of Lang and Daybell's work, the gas may be deliberately injected into the flow at selected sites on the surface of submerged bodies: this is called forced, selective, or artificial ventilation and this technique is a useful control device, as mentioned previously. Lifting surface-piercing foils are very susceptible to ventilation, which may be triggered by waves or other disturbances. The ventilated flow once formed may be quite stable and exhibit a large hysteresis persisting into a normally fully wetted domain. "Fences" are normally used to prevent access of surface air if natural ventilation is not wanted.

It is generally thought that a region of separated flow (on an initially fully wetted body) is required for the initiation of natural ventilation (Wetzel 1958). This is readily available on a bluff surface-piercing body such as a circular cylinder or blunt-based hydrofoil, and the extent beneath the free surface of such ventilation as a function of speed correlates with the preexisting fully wetted wake underpressure (in consistent units). This process is called creeping ventilation since it proceeds smoothly with flow speed. An example of this type of stable base ventilation is shown in Figure 5. At a larger angle of attack the foil becomes

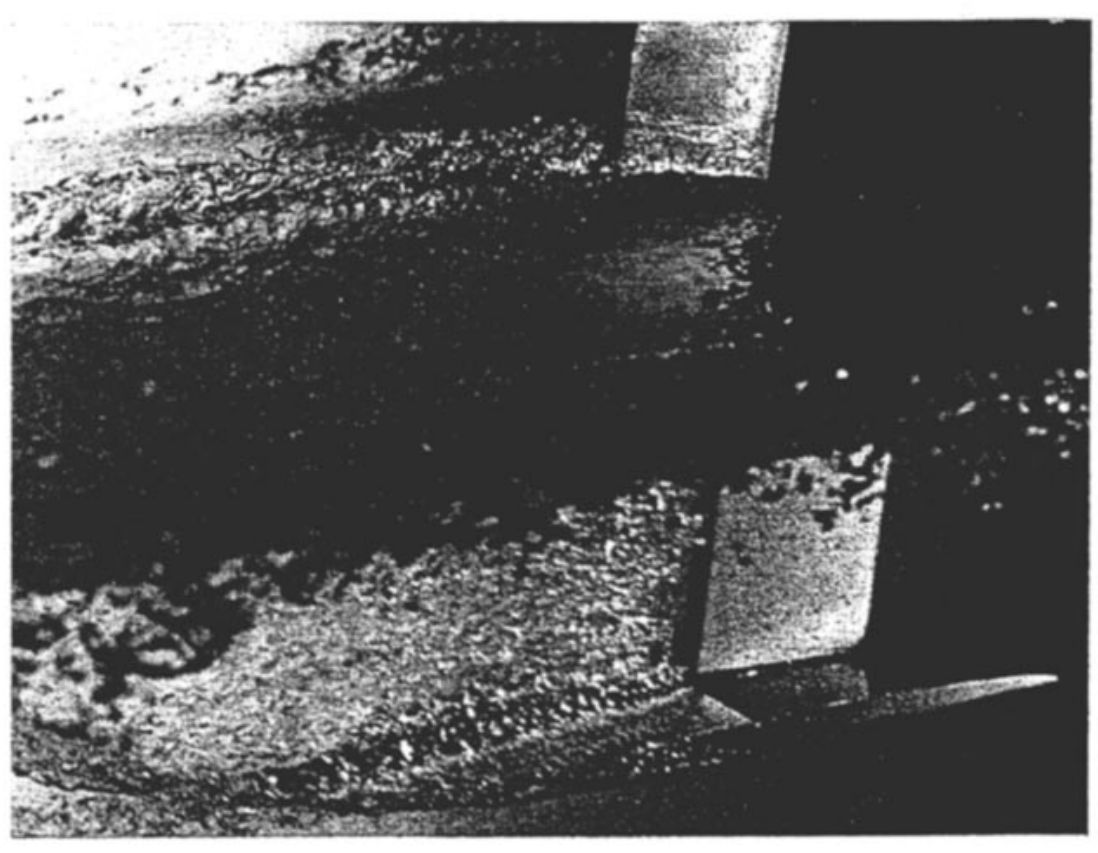

Figure 5 Photograph of cambered base-ventilating parabolic-wedge hydrofoil of aspect ratio unity supported on base-ventilating parabolic strut. The open cavity behind the strut provides the path of ventilating air from the surface to the hydrofoil. The flow speed is $18 \mathrm{ft} / \mathrm{sec}$ and the hydrofoil chord is 4 in. 


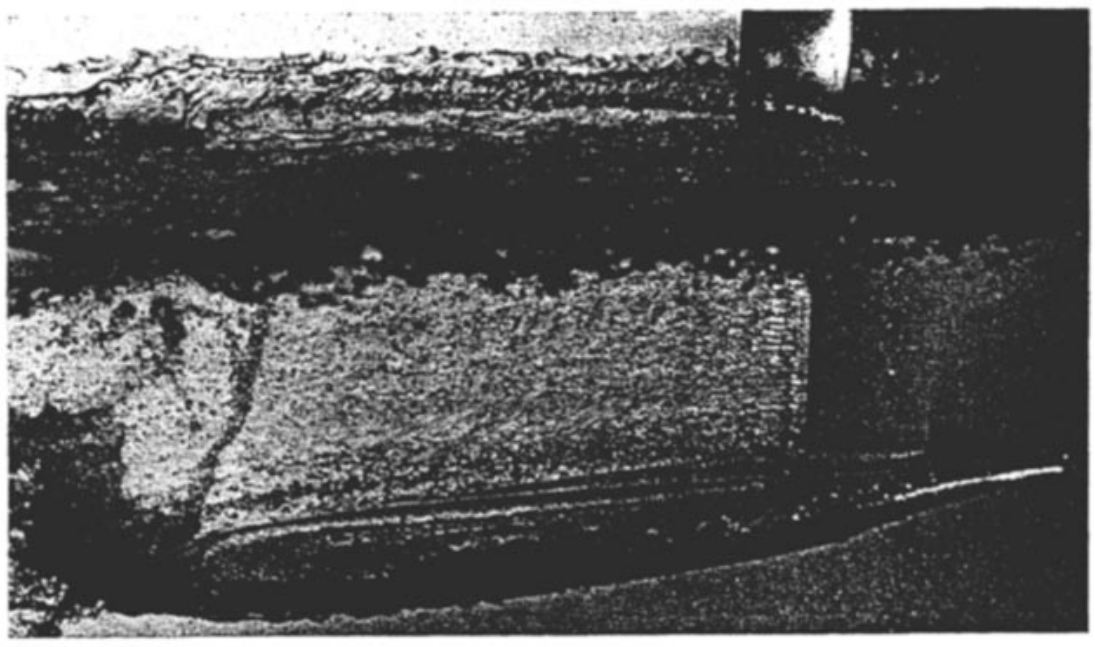

Figure 6 Photograph of the cambered hydrofoil in Figure 5 ventilated from the leading edge. The angle of attack is $10^{\circ}$ larger in this photograph.

spontaneously ventilated from the leading edge (Figure 6). However, when a separated low-pressure region has no ready access to the free surface, a major perturbation may be required to initiate ventilation; this case, highly speed sensitive, is termed flash ventilation and occurs on surface-piercing yawed struts because, as shown by Wadlin (1958), the lift falloff at the intersection with the free surface forestalls separated flow there for thin hydrofoils. The importance of locally separated flow as a condition for establishment of natural ventilation was highlighted by Wadlin (1958), who showed that surface-piercing foils could experience vapor cavitation without ventilating. Later Waid (1968) showed that these cavitating regions could themselves lead to surface ventilation. Another important route of natural ventilation is via the trailing vortex core system, which at high speed may communicate with the distorted free surface downstream of the foil and is cited by Parkinson (1956) as a major problem in application of hydrofoils to seaplanes. Figure 7 exhibits this type of ventilation on a delta wing.

The amount of air required to support forced ventilation and physical mechanisms responsible for air entrainment have received some attention; turbulent mixing at the cavity terminus, driven by the tendency to form an unstable reentrant jet, is responsible for entrainment in two-dimensional flows. The amount of air required increases with the length of the cavity, and roughly inversely with the cavitation number. It should be noted that the orientation of gravity is important in reentrant-jet mixing. Lifting surfaces form ventilated vortex cores through which (depending on surface proximity and Froude number) air may be 


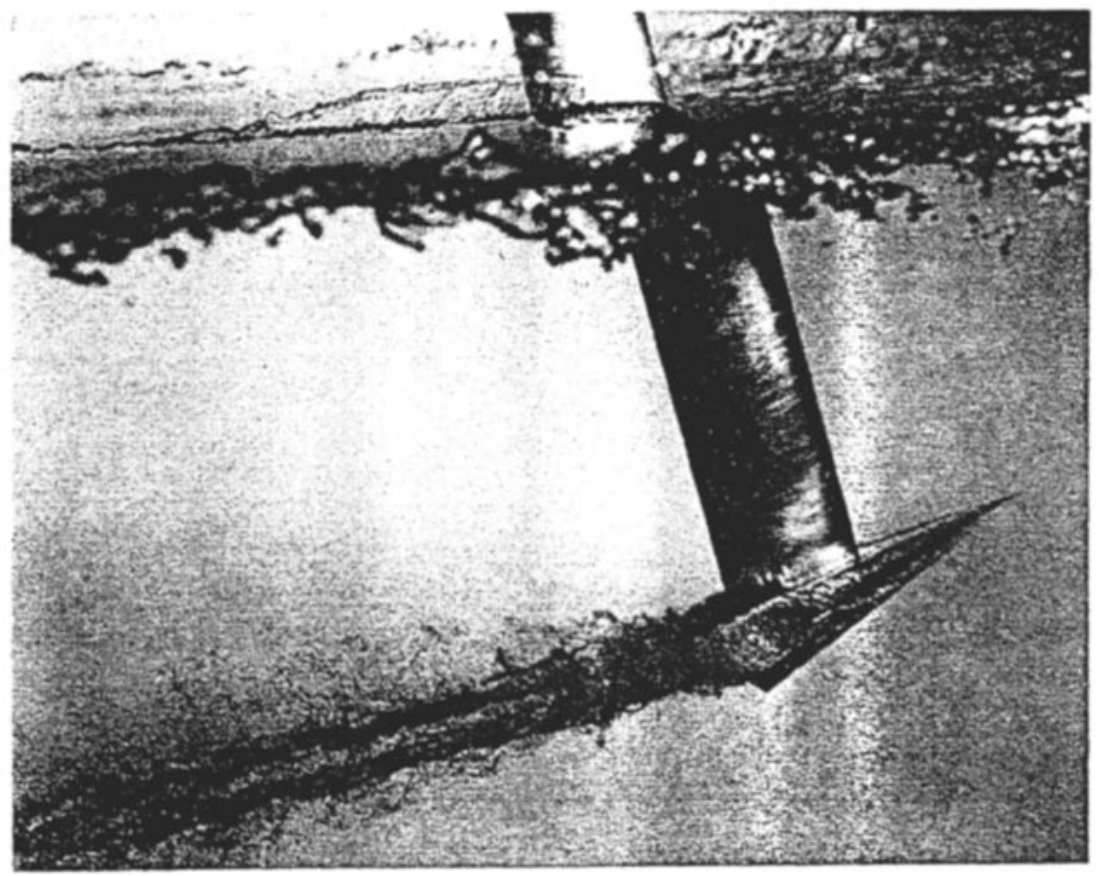

Figure 7 Ventilation on a $30^{\circ}$ apex angle delta wing at an angle of attack of $25^{\circ}$. The slender strut does not ventilate. The ventilated vortex cores disappear unless air is supplied either on the foil or from downstream.

lost instead of supplied (Schiebe \& Wetzel 1961). The transverse buoyancy effect on horizontal ventilated cavities behind deeply submerged nonlifting bluff bodies also leads to a Froude-number-dependent trailing-vortex pair (Campbell $\&$ Hilborne 1958), the required air flow then increasing sharply with the formation of these vortex tubes. As a general rule it may be said that any external disturbance such as unsteady motion of the foil or passage through waves tends to increase the air flow required (Klose \& Acosta 1969, Wetzel \& Foerster 1967), although the effect depends greatly upon the type of disturbance, its frequency, and its amplitude. A curious effect has been described by Song (1962), who noted that superventilated cavities in a gravity-free environment adjacent to a free surface could develop stable oscillations having a definite frequency for very large rates of air supply.

Selective ventilation in which gas is injected through orifices on the surface of a hydrofoil is described by Lang \& Daybell (1961). They point out that a stable cavity can be formed at various locations downstream of the leading edge if the liquid flow at the site of injection is unseparated or noncavitating, and they also provide a great wealth of information on flow rates, injection patterns, range of 
lift modulation, and change of lift slope to be expected. The ventilated flow state must become established or removed rapidly if useful dynamic control is to be achieved. This question is addressed experimentally by Acosta \& Wade (1967), who show that fully wetted flow can be reestablished in a time corresponding to the travel of a few chord lengths, provided the basic flow is not separated. The establishment of the forced-ventilation cavity in a normally noncavitating flow takes longer.

Steady superventilating and supercavitating flow past bodies with long cavities, as in Figure 6, are very similar, as then a free streamline of essentially constant pressure is the primary feature of importance; indeed, superventilation has proved to be a valuable means of exploring free streamline flows experimentally, provided the location of the cavity detachment points is known.

\section{HYDROFOILS}

We now consider briefly the flow past the hydrofoils themselves. In addition to the effects of the free surface, cavitation, and ventilation already mentioned, unsteady motions are particularly important in determining hydrodynamic response for stability and control and also in deducing the hydroelastic interactions that may lead to flutter or divergence. The field of unsteady hydrodynamics as applied to hydroelasticity has been recently reviewed by Abramson (1969) and more comprehensively in the useful earlier monograph by Abramson and coworkers (1967). We shall not pursue this area extensively except to note that the tools of analysis here as in low-speed unsteady aerodynamics are based largely on incompressible potential flow augmented, when required, by boundarylayer concepts. The additional problems encountered when cavitating or ventilating wakes are introduced into an unsteady flow are discussed from a theoretical point of view by Wu (1972). We may remark here that linearized theories play a much more important role in hydrofoil analysis than in corresponding aeronautical applications because of the complications afforded by the free surface and finite cavity wakes. It may be presumed therefore that many of these results are not as firmly based as one would like.

\section{The Free Surface}

Hydrofoils, unlike airfoils, have the problem of never being far away from a boundary, in the present case the air-water interface. Controlled by gravity, the displacement of this interface is unknown in advance and it constitutes a speedor more properly Froude-number-dependent constant-pressure surface that interacts with the local flow about the hydrofoil. Two limiting assumptions are often made in this context, zero Froude number and infinite Froude number. The first use corresponds to a rigid boundary, whereas in the second there is no effect of gravity at all; both are readily treated in linear theories by the method of images. Actual situations lie between these extremes, and because of the existence of waves originating from the disturbance a wavedrag becomes possible, a 
subject of central importance in naval hydrodynamics. The estimation of this wave resistance has long been a concern in the application of hydrofoils. Some of the earliest results for two-dimensional submerged fully wetted foils were given by Keldysh \& Lavrent'ev (1936) and Kochin (1937), who deduced the formula $C_{v} / C_{L}^{2}=\left(g c / 2 V^{2}\right) \exp \left(-2 g d / V^{2}\right)$ for the wavedrag coefficient $C_{v o}$. Here $C_{L}$ is the lift coefficient, $V$ the fluid speed, $g$ the acceleration of gravity, $c$ the chord, and $d$ the submergence. This result shows that the wave contribution to the drag-lift ratio of hydrofoil craft is quite small at typical speeds. The presence of the free surface modifies the two-dimensional sectional characteristics of hydrofoils in addition to creating wavedrag. A recent treatment due to Hough \& Moran (1969) is illustrative of the approach: thin hydrofoils are represented by a bound vortex distribution, the potential representing the vortex also satisfying the free-surface boundary condition for small water waves, $V^{2} u_{x}+g v=0$, where $(u, v)$ are the perturbed Cartesian velocity components. This leads to a Froudenumber-dependent distribution of doublets trailing downsteam from the vortex but located at the image point in the undisturbed free surface. The usual linearized-flow tangency conditions on the wetted foil surface are applied, to result in an airfoil-type integral equation for the bound vorticity distribution, which is solved by a collocation procedure. The gravity effect tends to reduce the lift coefficient as shown in Figure 8, and in two-dimensional flow a chord Froude number of 10 is required before the effect is negligible. An alternative and promising method of solving two-dimensional problems with greater precision is given by Giesing \& Smith (1967), who satisfy boundary conditions exactly on the wetted body by surface source distributions, each of which satisfies the linearized free-surface condition. The resulting integral equations are solved numerically.

The effect of finite aspect ratio on the characteristics of a fully wetted submerged hydrofoil with the gravity effect was first treated by Wu (1954), then later by Breslin (1957, 1961), by lifting-line techniques in which it is shown that the nonviscous drag may be considered to be composed of the induced drag of the wing in an unbounded fluid, the induced drag due to its biplane image in the free surface, and an additive wave resistance. This latter decays asymptotically as chord Froude number squared, so that this component of resistance may be neglected for chord Froude numbers beyond about 5 for a like aspect ratio. This problem has also been treated at length by Nishiyama (1965b), who also considers the effect of dihedral and surface-piercing foils. An "optimum" distribution of circulation is deduced which requires a constant induced velocity distribution, this, however, under the influence of the surface waves. The resulting circulation distribution is fuller than the elliptic one associated with unbounded flows. The effect of flaps or ailerons is also treated by the lifting-line approach (Nishiyama 1964); the lifting-line method is reconsidered by Nishiyama (1965a) with an approximate lifting-surface theory. These calculations are again based on linearized theory but show good agreement with experiment in its range of validity up to about $5^{\circ}$ in angle of attack. The lifting-line method is shown to overestimate the lift slope by about $25 \%$ at an aspect ratio of 4 . The gravity effect, however, diminishes with decreasing aspect ratio and for an aspect ratio of unity appears to be quite negligible for a chord-to-depth ratio of one-half. 


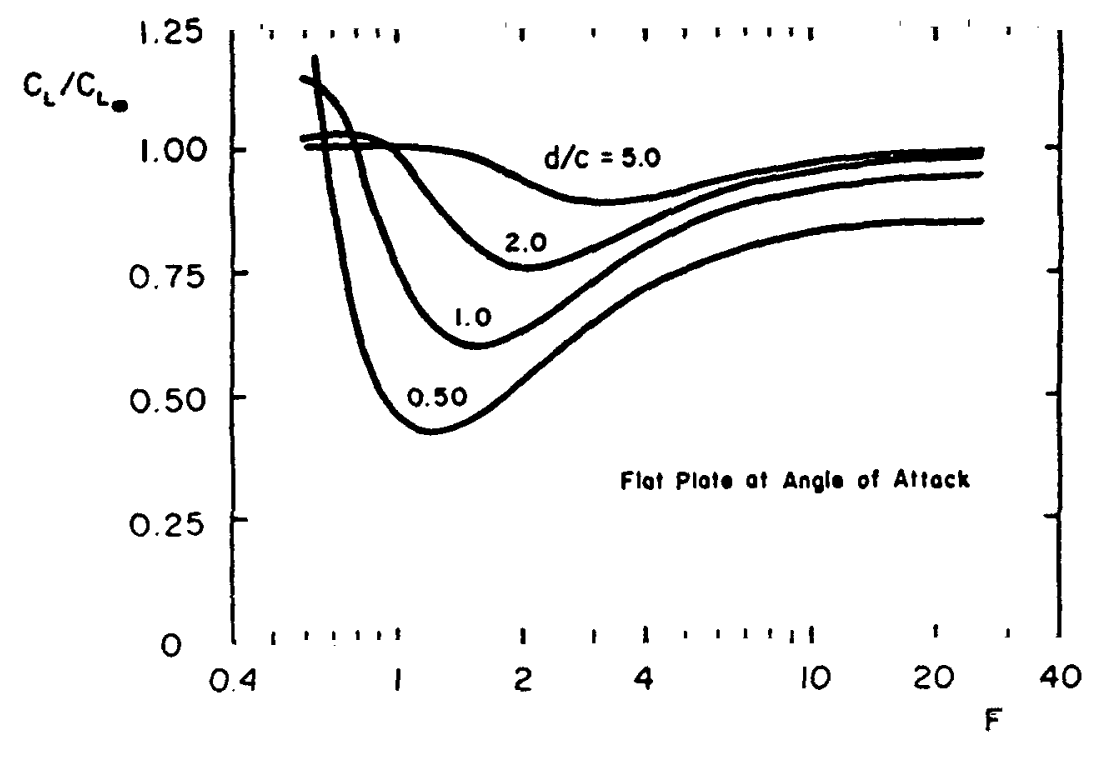

Figure 8 The effect of Froude number based on chord $F=V(g c)^{-1 / 2}$ and submergence $d$ beneath the surface on the lift of a fully wetted flat-plate hydrofoil, from Hough \& Moran (1969), $c$ being the chord and $d$ the submergence. (Courtesy Society Naval Architects and Marine Engineers, used by permission.)

[Somewhat similar results on the effect of Froude number for planing surfaces of finite-aspect ratio are also found by Wang \& Rispin (1971), where it is shown that above an aspect ratio of approximately unity, the gravity effect decreases lift, about unity the effect is null, and below unity lift is actually increased.] These results tend to justify the zero gravity assumption of ten made in submerged hydrofoil work. Although this may be permissible on a local basis, especially for low aspect ratios, surface distortions far downstream which may be of importance are very Froude-number dependent.

In the event that the zero-gravity approximation can be made, the submerged fully wetted hydrofoil becomes, in effect, a biplane insofar as the bound vortex distribution is concerned; the effects of taper, sweep, and planform variations can then be translated directly from aeronautical practice. Profile thickness, which in linear theories is represented by a source distribution, requires a negative image in the free surface. This is the basis of an important numerical method we shall discuss later in the context of supercavitating foils.

\section{Supercavitating Foils}

The loss of leading-edge suction in supercavitating flow leads to a pressure or form drag which precludes use of conventional subsonic airfoils in these applica- 
tions. A major advance was made by Tulin \& Burkart (1955), who pointed out that the wetted pressure surface of the foil could be designed in such a way as to optimize in a certain limited sense the lift-drag ratio; use of these concepts was greatly facilitated by the linearized free streamline developed by Tulin and recently reviewed by Wu (1972) (see also Tulin 1964). The wetted profiles considered in their optimization were of the form

$$
\eta=\sum_{1}^{n} a_{n}(x)^{(n+1) / 2}
$$

$\eta$ being the dimensionless ordinate and $x$ the chord. Originally, Tulin and Burkart considered two terms, later Johnson (1961) used up to five terms. The coefficients $a_{n}$ were determined with the linear theory at zero cavitation number in such a way that the pressure on the wetted surface never became negative. Under ideal conditions the loading of these optimum foils peaks near the trailing edge with smooth entry at the leading edge; the theoretical two-dimensional liftdrag ratios are found to be large at this ideal condition $\left(L / D=17.5 / C_{L}, n=5\right)$. However, these ideal conditions are not realizable physically because of the necessity for leading-edge mechanical strength and because of the requirement that the free streamline miss the foil surface. This latter condition may be achieved by operating the foil at a higher angle of attack and/or providing some bluntness to the nose to provide strength as well as to cause the cavity to spring clear.

Marine propellers are particularly well suited for the application of supercavitating foils. Tulin (1962), in a very interesting review, summarizes early developments in cavitating propellers and the development of suitable supercavitating profiles for them. Later B. R. Parkin (1964), using an inverse procedure with the linear theory, discusses the design of optimum profiles at nonzero cavitation numbers with sufficient room for the cavity to clear the end of the wetted trailing edge; $20 \%$ of the chord is recommended. Under these conditions, if a three-term profile is used, the lift-drag ratio is found to be only 7.85 with a lift coefficient of $C_{L}=0.4$, which although not particularly high may be more realistic. More recently, Larock \& Street (1968) have produced the basis for a design procedure with an exact free-streamline theory; as an example they exhibit a profile having a lift-drag ratio of 11.1 with $C_{L}=0.4$ with a cavity clearance of $17 \%$ at the end of the foil. Murai \& Kinoe (1968) develop an exact method somewhat similar to that described by Wu (1972) for zero cavitation number. They carry out analyses of three-term hydrofoils with blunted noses; for a design lift coefficient of 0.4 they report lift-drag ratios of 9.25 with zero leading-edge radius decreasing by $7 \%$ for $0.3 \%$ chord (approximately) radius. Several supercavitating profiles are gathered together in Figure 9 to illustrate these features. It may be remarked that good use of the cavity space has been made in the "Hydronautics" profile by adding a strength member that can also be used for control purposes (Oakley 1963).

The two-dimensional characteristics of supercavitating foils are greatly modified by the presence of the free surface (we now neglect the effect of gravity), 

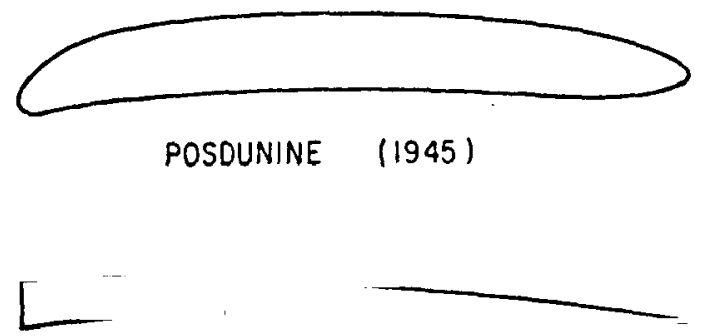

TMB-TULIN (1954)
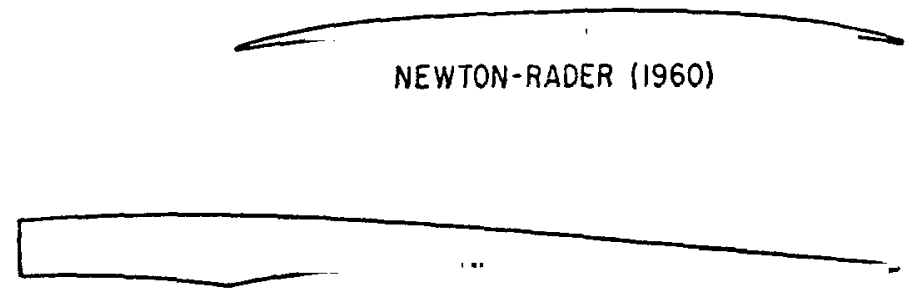

HYDRONAUTICS (1961)

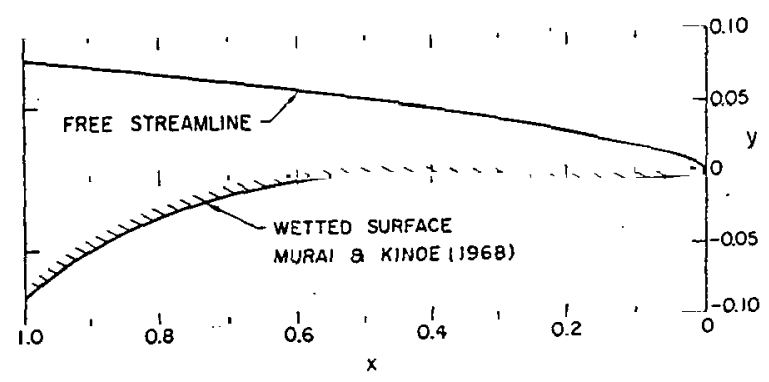

Figure 9 Supercavitating hydrofoil profiles after Tulin (1962), Murai \& Kinoe (1968). The latter two profiles have blunted noses needed for strength. The flow is from right to left and normally the upper surface of the profiles is in the vapor cavity.

although the effect of aspect ratio acts to diminish this change. The lift slope of a two-dimensional supercavitating flat plate hydrofoil at zero cavitation number has the at-first surprising behavior of increasing from $\pi / 2$, deeply submerged, to $\pi$ at the surface, which is precisely contrary to the behavior of fully wetted hydrofoils. This surface effect has been widely studied using the linear theory, by Johnson (1961) and Auslaender (1962), for example, and by Larock \& Street (1967), who carry out an exact hodograph calculation for a flat plate. Cambered foils 
show a less extreme behavior-for example, a circular arc at zero angle of attack exhibits about a $20 \%$ lift increase, with the previously mentioned five-term foil about half this (Johnson 1961). Nevertheless, the predominant effect of the surface in supercavitating flow is to augment rather than decrease lift, which is a destabilizing effect for a craft.

\section{Finite Aspect Ratio}

Although considerable theoretical guidance for the development of two-dimensional supercavitating foils became available through the work of Tulin and then $\mathrm{Wu}$, the three-dimensional effects of finite aspect ratio pose a problem of a much greater level of difficulty. This is in part because the convenient means of analysis in two-dimensional potential flow are not available; it is also due to the unknown configuration of the three-dimensional constant pressure boundary (this being circumvented to some extent in two dimensions with the hodograph transformation). Except in certain simplified cases (to be discussed), it is difficult to proceed with any numerical method except in a rough way unless the general features of the flow boundary are known to begin with. Figures 6 and 7 illustrate the problem. Figure 6 is a foil ventilated from the leading edge and shows that the ventilation cavity is more or less two dimensional in conformation, except possibly at the tips. Figure 7, however, shows a strongly three-dimensional conical tip cavity. Close inspection reveals that the foil is wetted between these ventilated cavities. Observations have been made on rectangular supercavitating foils (Kermeen 1961 and Spengler 1966) which show similar tip cavities at moderate angles of attack with triangular wetted portions on the upper foil between this cavity and the inboard stations, which exhibit two-dimensional cavitating patterns. For sufficiently large angles of attack, depending upon aspect ratio and depth, all these flows become fully cavitating as in Figure 6 .

Different theories have been put forth to explain these several flow configurations. One of the first was by Johnson (1961), who ingeniously combined results from the linear and nonlinear theories aleady mentioned for zero cavitation number and arbitrary camber lines, together with the depth effect previously discussed, with finite-aspect-ratio corrections borrowed directly from smallaspect-ratio lifting-surface aerodynamics. For the conditions intended, fully ventilated foils at zero cavitation number, this engineering procedure works very well. Johnson also made estimates of the free-streamline position above the wetted foil to determine bounds on the lift coefficient which could not result in interference with the foil; on this basis a maximum lift-drag ratio of about 10 may be achieved at a lift coefficient of $C_{L}=0.2$. The subsequent experiments of Spengler and Dobay on aspect-ratio-3 foils indicate that somewhat poorer liftdrag ratios are obtained than theory would indicate, but the experimental conditions are not sufficiently well defined to determine the cause of the difference. A completely different approach is taken by Widnall (1966), Nishiyama \& Miyamoto (1969), and Nishiyama (1970), who use the fully linearized, threedimensional acceleration potential. In all three of these works, pressure doublets 
are used to represent the wetted surface and pressure sources to represent the cavity. These distributions are applied on the central plane of the hydrofoil cavity system, just as in the corresponding two-dimensional linear theory. There are some differences between these methods in the treatment of the cavity length but the main difference is in the method of satisfying the resulting integral equations for the unknown strengths of the singularity distributions. Widnall uses a numerical treatment based on control points; Nishiyama ultimately makes a lifting-line approximation; whereas Nishiyama and Miyamoto carry out a liftingsurface approximation based on the known two-dimensional supercavitating singularity distributions. These results are, on the whole, comparable and agree reasonably with experiments on a supercavitating hydrofoil with an aspect ratio of 6. Widnall, in addition, considers unsteady motions with the acceleration potential and Nishiyama (1969) takes into account the free-surface effect (neglecting gravity) by the image method.

Several conclusions can be drawn from these findings: the free-surface augmentation effect is considerably diminished as is the effect of aspect ratio on reducing lift, and the spanwise lift distribution is flatter than in fully wetted flow. On the whole these trends have also been found experimentally but there are difficulties with the tips and it is not clear if these procedures can be extended to lower aspect ratios or into nonlinear regions. A rather different approach is taken by Cumberbatch (1961), who models a quasi-slender-body theory for rectangular low-aspect-ratio hydrofoils exhibiting the conical tip cavity already mentioned. This approach agrees very well with experiment for aspect ratios of 1 and 2 even for large angles of attack, but for an aspect ratio of 4 all these procedures tend to overestimate the lift.

Supercavitating delta wings have also received attention. At moderate angles of attack partial ventilation or cavitation occurs, as in Figure 7. This configuration has been analyzed with a conical flow approach by Tulin (1959) and later, with some modification, by Kaplan, Goodman \& Chen (1967); the agreement with experiment is quite satisfactory up to apex angles of $45^{\circ}$ even with angles of attack up to about $15^{\circ}$. For greater angles of attack the flow becomes fully cavitating, i.e., the cavity does not contact the upper profile. This configuration is treated successfully by Cumberbatch \& Wu (1961), who use a slender-body analysis. The delta-wing configuration would not be considered as a primary lifting-surface element on a hydrofoil craft because of its comparatively large induced drag; nevertheless it may well serve in some auxiliary capacity.

\section{Base Ventilating Foils}

Comparatively thick truncated foils as shown in Figure 5 can be used to advantage in ventilated flow if the base pressure is near ambient. The pressure-drag coefficient is then comparatively small $\left(C_{D}=0.394 \epsilon^{2}\right.$ for a parabolic wedge based on planform area, $\epsilon$ being the thickness ratio) and the usefulness in the concept is that the forebody is under a positive pressure and has a large section modulus. In addition, camber can be superposed to develop lift, provided the minimum 
pressure so obtained is somewhat larger than the vapor pressure. Johnson \& Rasnick (1959) have made a detailed study of this concept as have Lang \& Daybell (1961), who also provide supporting experimental evidence of good lift-drag ratios. A foil system based on the base ventilated principle was tested at speeds up to 80 knots (Ellsworth 1967). At this speed and certainly for higher speeds the chance possibility of cavitation is very likely. Base ventilation therefore appears to fulfill a role intermediate in speed between fully wetted and supercavitating flows. One application of this type of hydrofoil has been on the Bras $D^{\prime} O r$, already mentioned.

\section{SUMMARY-PROSPECTS}

Up to a speed of about 50 knots, hydrofoil systems can be designed to operate without significant cavitation effects. Present hydrodynamic theories are sufficient for calculation of forces and other overall quantities in this speed range except possibly for details such as pressure distributions, etc, where further work appears to be needed. Ventilation of surface-piercing components is a problem that, although imperfectly understood, appears to be coped with on a practical basis. It would seem reasonable therefore to expect continued development of large craft in this speed range provided there is a naval or commercial requirement.

If an adquate state-of-the-art technology may be said to exist in the 50-knot range, there is only a basis for such a technology at higher speeds, say 70 knots and up. In this range of speeds the first recourse must still be the laboratory to define the interactions between cavitation, ventilation, and the free surface and mutual effects between bodies and unsteady states of flow, and the response of the associated structures. Additional experimental work of both an applied and a basic nature is required to establish proper physical models. Beyond this, research on effective means for the calculation of three-dimensional flows including cavitation/ventilation in the presence of the free surface would be very beneficial. Perhaps then, with parallel advances in propulsion, it may be possible to determine if, after all, a truly high-speed hydrofoil-craft technology will emerge.

\section{ACKNOWLEDGMENTS}

I would like to acknowledge many helpful discussions with Mr. William O'Neill and Mr. Alec Silverleaf. I would also like to thank the Office of Naval Research, Fluid Mechanics Branch, for their continued support who, together with the Naval Ship Research and Development Center, have made the preparation of this article possible. 


\section{Literature Cited}

Abramson, H. N. 1969. Appl. Mech. Reo. 22:115-21

Abramson, H. N., Chu, W. H., Irick, J. T. 1967. Hydroelasticity with special reference to hydrofoil craft, US Naval Ship Res. \& Develop. Ctr., Rep. 2557

Acosta, A. J., Wade, R. B. 1967. Selectively ventilated ring wing hydrofoils, California Inst. Technol., Rep. E-138.3, Pasadena, Calif.

Anon., 1958. Study of Hydrofoil Seacraft, V. I, II, Grumman Aircraft Rep. Bethpage, New York

Arakeri, V., 1971. Water tunnel investigations of scale effects in cavitation detachment from smooth slender bodies and characteristics of flow past a bi-convex hydrofoil, California Inst. Technol., Rep. E-79A.12, Pasadena, Calif.

Auslaender, J. 1962. J. Ship Res. 6, no. 2:8-23

Breslin, J. P. 1957. J. Ship Res. 1, no. 1: $27-35,55$

Breslin, J. P. 1961. J. Stip Res. 5, no. 2: 15-21

Breslin, J. P., Landweber, L. 1961. Soc. Nav. Arch. Mar. Eng. Tech. Res. Bull., No. $1-21$. iv +53 pp. +25 figs.

Buermann, T. M., Leehey, P., Stillwell, J. J. 1953. Trans. Soc. Nav. Arch. Mar. Eng. 61:242-64; disc. 265-79

Campbell, I. J., Hilborne, D. V. 1958. In Second Symp. Nav. Hydrodynam., ed. R. D. Cooper, 467-81

Cooper, R. D., Ed. 1958. Second Symp. Hydrodynam., Washington, DC

Crewe, P. R. 1958. Trans. Inst. Nao. Arch. 100:329-62; disc. 362-73

Cumberbatch, E. 1961.J. Ship Res. 4, no. $4: 1-8$

Cumberbatch, E., Wu, T. Y. 1961. J. Fluid Mech. 11:187-208

Dobay, G. F. 1965. Hydrofoils designed for surface ventilation, Soc. Nav. Arch. Mar. Eng. Hydrofoil Symp., Seattle, paper no. 2f. 12 pp.

Dobay, G. F., 1967. Influence of scale ratio, aspect ratio and planform on the performance of supercaditating hydrofoils, Naval Ship Res. Develop. Ctr., Washington, DC. Rep. 2390 . vi +79 pp.

Eames, M. C., Jones, E. A. 1970. Hover. Craft Hydrofoil 9, no. 8:8-20

Egorov, I. T., Sadovnikov, Yu. M., Isaev, I., Basin, M. A. 1971. Iskusstoennaya kavitatsiya (Artificial Cavitation). Leningrad: Sudostroenie. $283 \mathrm{pp}$.

Eisenberg, P., Tulin, M. P. 1961. In Handbook of Fluid Dynamics, ed. V. E.
Streeter, Sect. 12. New York: McGrawHill

Ellsworth, W. M. 1967. The U. S. Nady Hydrofoil Development Program-A Status Report. AIAA/SNAME Ado. Mar. Veh. Symp.

French, H. R., Holgate, T. N., Jamieson, J. J. 1962. Proc. Hydrofoil Air Cush. Veh., 23-34. Inst. Aerosp. Sci.

Gabrielli, G., von Kármán, T. 1950. Mech. Eng. 72:775-81

Geyer, L. A., Wennagel, G. J. 1959. Trans. Soc. Nao, Arch. Mar. Eng. 67: 686-706; disc. 706-14

Giesing, J. P., Smith, A. M. O. 1967. J. Fluid Mech. 28:113-29

Green, T., Street, J. L. 1967. J. Fluid Mech. 27:1-28

Hirsch, I. A. 1967. On the Prediction of the Sea Keeping Characteristics of Hydrofoil Ships. AIAA/SNAME Ado. Mar. Veh. Symp.

Hough, G. R., Moran, J. P. 1969. J. Ship Res. 13:53-60

Johnson, V. E., Jr. 1961. Nat. Aero. Space Adm. Tech, Rept. R-93, $78 \mathrm{pp}$.

Johnson, V. E., Jr., Rasnick, T. A. 1959. Nat. Aero. Space Adm. Tech. Note $D-119,27 \mathrm{pp}$.

Kaplan, R., Goodman, T. R., Chen, C. C. 1967. J. Hydronout. 1:81-88

Keldysh, M. V., Lavrent'ev, M. A. 1936. On the motion of a wing under the surface of a heavy fluid. In Trudy Konferentsit po Teorii Volnovogo Soprotideniya, Moscow, 1936, 31-64. Moscow: Tsentral. Aero-Gidrodinam. Inst. [Engl. transl.: Sci. Trans. Service STS-75, Cambridge, Mass., 1949]

Kermeen, R. W. 1956. Water tunnel tests of N.A.C.A. 4412 and Walchner hydrofoils in non-cavitating and cavitating flows, California Inst. Technol. Rep. 47.5, Pasadena, Calif.

Kermeen, R. W. 1961. J. Ship Res. 5, no. $2: 22-43$

King, D. A., Land, N. B. 1952. Nat. Adv. Comm. Aero. Rep. L52JIO

Klose, G. J., Acosta, A. J. 1969. J. Ship Res. 13:92-102

Knapp, R. T., Daily, J. W., Hammitt, F. G. 1970. Cavitation. New York: McGraw-Hill

Kochin, N. E. 1936. On the wave resistance and lift of bodies submerged in a fluid, In Trudy Konferentsii po Teorii Volnovogo Soprotioleniya, Moscow, 1936. Moscow: Tsentral. Aero-Gidrodinam. 
Inst. [Engl. transl.: SNAME Tech. Res. Bull. No. 1-8, 1951]

Krack, R. C., Gross, J. G. 1965. Experience with the hydrofoil craft Denison. Soc. Nav. Arch. Mar. Eng. Hydrofoil Symp., Seattle, paper no. 2e. 27 pp.

Lang, T. G., Daybell, D. A. 1961. J. Ship Res. 5, no. $3: 1-15$

Larock, B. E., Street, R. L. 1967. J. Ship Res. 11:131-9

Larock, B. E., Street, R. L. 1968. J. Ship Res. 12:1-13

McLeavy, R., Ed. 1971. Jane's Surface Skimmers. London: Paulton

Martin, M. 1967. Hydrofoil craft dynamics in a realistic sea including automatic control, Hydronautics Inc., Laurel, Md., Rep. 463-10

Murai, H., Kinoe, T. 1968. Sci. Rep. Res. Inst. Tohoku Univ. Ser. B. Rep. Inst. High Speed Mech. 20:263-87

Nishiyama, T. 1964. Schiffsteclunik 11: 72-6

Nishiyama, T. 1965a. J. Ship Res. 8, no. $4: 1-11$

Nishiyama, T, 1965b. J. Ship Res. 9:131-2

Nishiyama, T. 1970. Z. Angew. Math. Mech. 50:645-53

Nishiyama, T., Miyamoto, M. 1969. Technol. Rep. Tohoku Unio. 34:327-43

Numachi, F. 1964. Trans. ASME, J. Basic Eng. 86: 543-55

Numachi, F., Nakamura, M., Chida, I. 1959. Sci. Rep. Res. Inst. Tohoku Unio. Ser. B. Rep. Inst. High Speed Mech. 10:41-128

Oakley, O. H. 1963. Hover. Craft Hydrofoil 2 , no. $5: 14-26$

Parkin, B. R. 1964. Trans. ASME, J. Basic Eng. 86:641-55

Parkin, B. R., Kermeen, R. W. 1957. J. Ship Res. 1, no. 1:36-42

Parkin, B. R., Peebles, G. H. 1956. Soc. Nav. Arch. Mar. Eng. Tech. and Res. Bull. No. 1-17. 55 pp.

Parkin, J. H. 1964. Bell and Baldwin. Toronto: Univ. of Toronto Press

Parkinson, J. B. 1956. First Symp. Nav. Hydrodynam., ed. F. S. Sherman, 181213

Robertson, J. W., Wislicenus, G., Eds. 1969. Cavitation State of Knowledge. New York: Am. Soc. Mech. Eng.

Schiebe, F. R., Wetzel, J. M. 1961. Ventilated carities on submerged 3-dimensional hydrofoils, Univ. Minn. St. Anthony Falls Hydraul. Lab. Tech. Pap. No. 36

Schultz, W., Coffey, R. J., Gornstein, R. J. 1971. High Speed Water Transportation of Man, ASCE/ASME, Nat. Transp. Eng. Mtg.
Schuster, S., Schwaneke, H. 1960. In Third Symp. Nav. Hydrodynam., ed. S. W. Doroff. 147-89

Silverleaf, A. 1969. Inst. Mech. Eng. Proc. $184: 1221-44$

Silverleaf, A., Cook, F. G. R. 1970. Trans. Inst. Nav. Arch., 112:69-78; disc. 78-86 Smith, K. E. 1963. US Patent 3,096,739

Song, C. S. 1962.J. Ship Res. 5, no. 4:8-20

Spengler, P. K. 1966. David Taylor Model Basin Rep. 2353

Tulin, M. P. 1959. J. Ship Res. 3, no. 3: 17- 22

Tulin, M. P. 1960. In Third Symp. Nao. Hydrodynam., ed. S. W. Doroff, 121-45

Tulin, M. P. 1962 In Fourth Symp. Nao. Hydrodynam., ed. B. L. Silverstein, 239-86

Tulin, M. P. 1964. J. Ship Res. 7, no. 3: 16-37

Tulin, M. P., Burkart, M. P. 1955. David Taylor Model Basin Rep. C-638. iv +35 pp.

von Schertel, H. 1960. Third Symp. Nao. Hydrodynam., ed. S. W. Doroff, 233-69

von Schertel, H. 1963. Hocer. Craft Hydro foil 2, no. 6:10-12

von Schertel, H. 1970. Hocer. Craft Hydrofoil 9 , no. 7:7-13

von Schertel, H. 1972. Hooer. Craft Hydrofoil 11 , no. 4:4-7

Wade, R. B., Acosta, A. J. 1966. Trants. ASME, J. Basic Eng. 88:273-83

Wade, R. B., Acosta, A. J. 1967. Trans. ASME, J. Basic Eng. 89:693-706

Wadlin, K. L. 1958. Second Symp. Nao. Hydrodynam., ed. R. Cooper, 425-45

Waid, R. L. 1968. Experimental investigation of the ventilation of surface piercing struts in the presence of caoitation, Lockheed Missiles \& Space Co. Rep. DO 19597, Sunnyvale, Calif.

Walchner, O. 1932. Profilmessungen bei Kavitation. In Hydromechanische Probleme des Schiffsantriebs, ed. G. Kempf \& E. Foerster, 256-67. Hamburg

Wang, D. P., Rispin, P. 1971. J. Ship Res. 15:221-31'

Wetzel, J. M. 1958. Second Symp. Nao. Hydrodynam., ed. R. Cooper, 447-65

Wetzel, J. M., Foerster, K. E. 1967. Unsteady force and cavity characteristics for oentilated hydrofoils, Univ. of Minn. St. Anthony Falls Hydraul. Lab. Prog. Rep. No. 85. $x+38$ pp.

Widnall, S. E. 1966. J. Ship Res. 10:107-18

Wu, T. Y. 1954. J. Math. Phys. 33: 20748

Wu, T. Y. 1972. Ann. Reo. Fluid Mech. 4: 243-284 


\section{CONTENTS}

Ludwio Prandth in the Nineteen-Thrmies: Reminiscences, Irmgard Flügge-Lotz and Wilhelm Flügge

Use of Lasers for local Measurement of VeloctTy Components, Species Denstries, and Temperatures, S. S. Penner and T. Jerskey

EXPERIMENTS IN GASDYNAMICS OF EXPLOSIONS, $A$. $K$. Oppenheim and $R . I$. Soloukhin

Longtrudinal Dispersion and Turbulent Mixing in Open-Channel FLow, Hugo B. Fischer

Spherical-Cap Bubbles, Peter P. Wegener and Jean-Yoes Parlange

INTERMITTENCY IN LARGE-SCALR TURBULENT Flows, Erik Mollo-Christensen

Transonic Airfoils: Recent DeVelopments in Theory, Experdment, AND Design, G. Y. Nieuwland and B. M. Spee

Buoyant Plumes and Wakes, James A. Fay 151

Hydrofolls and Hydrofoll Craft, $A$. J. Acosta 161

The Flum Mechanics of Lugrication, E. A. Saibel and $N$. A. Macken 185

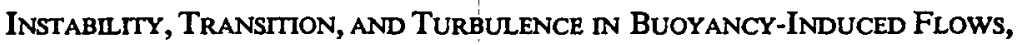
B. Gebhart

Secondary Flows: Theory, Experiment, and Application in TurboMACHINERY AERODYNAMICS, J. H. Horlock and B. Lakshminarayana

NoISE FROM AIRCRAfT TURBomachINERY, James E. McCune and Jack $L$. Kerrebrock

MdXing-Controlled Supersonic Combustion, Antonio Ferri 301

Three-Dimensional Boundary Layers, E. A Eichelbrenner 339

Hydrodynamic Flow Visualization, $H$. Werlé 361

Molecular Gas Dynamics, $M$. N. Kogan 383

PrandtL's Boundary-Layer Theory From the Viewpoint of a MatheMATICLAN, Karl Nickel 405

REPRINT INFORMATION $\quad 429$

INDEXES

AUTHOR INDEX

Cumulattve Index of Contributtang Authors, Volumes 1-5 439

Cumulative IndeX of Chapter Trthes, Volumes 1-5 440 